\title{
Respiratory variations of inferior vena cava fail to predict fluid responsiveness in mechanically ventilated patients with isolated left ventricular dysfunction
}

Hongmin Zhang ${ }^{1}$, Qing Zhang ${ }^{1}$, Xiukai Chen ${ }^{2}$, Xiaoting Wang ${ }^{1}$, Dawei Liu ${ }^{3 *}$ and Chinese Critical Ultrasound Study Group (CCUSG)

\begin{abstract}
Background: Respiratory variation of inferior vena cava is problematic in predicting fluid responsiveness in patients with right ventricular dysfunction. However, its effectiveness in patients with isolated left ventricular systolic dysfunction (ILVD) has not been reported. We aimed to explore whether inferior vena cava diameter distensibility index (dIVC) can predict fluid responsiveness in mechanically ventilated ILVD patients.

Methods: Patients admitted to the intensive care unit who were on controlled mechanical ventilation and in need of a fluid responsiveness assessment were screened for enrolment. Several echocardiographic parameters, including dIVC, tricuspid annular plane systolic excursion (TAPSE), left ventricular ejection fraction (LVEF), and LV outflow tract velocity-time integral (VTI) before and after passive leg raising (PLR) were collected. Patients with LV systolic dysfunction only (TAPSE $\geq 16 \mathrm{~mm}$, LVEF <50\%) were considered to have isolated left ventricular systolic dysfunction (ILVD).

Results: One hundred and twenty-nine subjects were enrolled in this study, among them, 28 were labelled ILVD patients, and the remaining 101 were patients with normal LV function (NLVF). The value of dIVC in ILVD patients was as high as that in NLVF patients, (20\% vs. $16 \%, p=0.211)$. The ILVD group contained a much lower proportion of PLR responders than NLVF patients did (17.9\% vs. 53.2\%, $p<0.001)$. No correlation was detected between dIVC and $\triangle V T I$ in ILVD patients $(r=0.196, p=0.309)$. dIVC was correlated with $\triangle V T I$ in NLVF patients $(r=0.722, p<0.001)$, and the correlation was strengthened compared with that derived from all patients $(p=0.020)$. A receiver-operating characteristic (ROC) analysis showed that the area-under-the-curve (AUC) of dIVC for determining fluid responsiveness from ILVD patients was not statistically significant $(p=0.251)$. In NLVF patients, ROC analysis revealed an AUC of $0.918(95 \% \mathrm{Cl}$ $0.858-0.978 ; p<0.001)$, which was higher than the AUC derived from all patients $(p=0.033)$. Patients with LVEF below $40 \%$ had a lower $\triangle V T I$ and fewer PLR responders than those with LVEF $40-50 \%$ and LVEF above $50 \%(p<0.001)$.
\end{abstract}

Conclusion: dIVC should be used with caution when critically ill patients on controlled mechanical ventilation display normal right ventricular function in combination with abnormal left ventricular systolic function.

Keywords: Echocardiography, Inferior vena cava, Fluid responsiveness, Heart function, Critically ill

\footnotetext{
*Correspondence: dwliu2015@sina.com

${ }^{3}$ Department of Critical Care Medicine, Peking Union Medical College

Hospital, Chinese Academy of Medical Sciences, 1 \# Shuai Fu Yuan, Dong

Cheng District, Beijing 100730, China

Full list of author information is available at the end of the article
}

\section{Springer Open}

(c) The Author(s) 2019. This article is distributed under the terms of the Creative Commons Attribution 4.0 International License (http://creativecommons.org/licenses/by/4.0/), which permits unrestricted use, distribution, and reproduction in any medium, provided you give appropriate credit to the original author(s) and the source, provide a link to the Creative Commons license, and indicate if changes were made. 


\section{Background}

Only $50 \%$ of haemodynamically unstable critically ill patients are fluid responders, and volume overload is detrimental to nonresponders; therefore, fluid responsiveness is frequently assessed in daily practice in the intensive care unit (ICU) [1, 2]. The diameter of the inferior vena cava (IVC) and the variation in this quantity are determined by the circulating blood volume and right ventricular (RV) function. For ICU patients on controlled mechanical ventilation, the IVC distensibility index (dIVC) was shown by previous studies to be a reliable indicator of fluid responsiveness [3, 4]. In contrast, some researchers have drawn different conclusions, and even the results of several meta-analyses about dIVC have been in-homogeneous $[5,6]$.

IVC diameters vary with the intra-abdominal pressure change during the respiratory cycle. In a number of clinical contexts, dIVC may not accurately predict fluid responsiveness. Prior studies have identified a relatively low tidal volume or a high PEEP as reasons for the inaccuracy of measuring fluid responsiveness from the IVC [7]. Another important factor is abdominal hypertension. Elevated intra-abdominal pressure reduces IVC size regardless of volume status, and is likely to affect the reliability of IVC-based fluid responsiveness assessment $[8$, 9].

RV dysfunction, including chronic pulmonary hypertension or RV myocardial infarction or severe tricuspid regurgitation, leads to significantly increased IVC size and reduced variation. Therefore, in the presence of RV dysfunction, the fluid responsiveness also cannot be reflected from IVC $[10,11]$. By contrast, if there is isolated left ventricular systolic function (ILVD), i.e., the RV function remains normal and the left ventricular (LV) systolic function is impaired, can IVC variation still be reflective of fluid responsiveness? ILVD is not a rare phenomenon in critically ill and is deemed an important mechanism of pulmonary oedema [12]. To the best of our knowledge, whether ILVD could influence the accuracy of dIVC has not been investigated. We hypothesized that when RV function is normal, while LV systolic function is impaired for various reasons, the IVC variation might not reflect fluid responsiveness. We thus performed this study to explore whether ILVD would affect the effectiveness of dIVC in assessing fluid responsiveness in mechanically ventilated critically ill patients.

\section{Patients and methods}

\section{Study population}

Patients admitted to the Peking Union Medical College Hospital ICU from 1 July 2018 to 1 January 2019 were screened for enrolment within the first $24 \mathrm{~h}$ after admission.
The inclusion criteria were as follows: mechanical ventilation without spontaneous breathing effort and the need for an assessment of fluid responsiveness due to hypotension, tachycardia, oliguria, and hyperlactatemia (with the decision to assess fluid responsiveness made at the discretion of an attending physician).

Patients with the following conditions were excluded from the study: RV dilatation and paradoxical septal motion, or tricuspid annular plane systolic excursion (TAPSE) below $16 \mathrm{~mm}[13,14]$; rhythm characteristic of atrial fibrillation; valvular diseases such as severe mitral, aortic or tricuspid stenosis or regurgitation; left ventricular outflow tract (LVOT) obstruction that was diagnosed by a high-velocity, late-peaking, dagger-shaped continuous-wave Doppler signal in conjunction with a peak gradient of at least $30 \mathrm{mmHg}$ [15]; intra-abdominal pressure equal or above $12 \mathrm{mmHg}$ [16]; an inadequate echocardiographic image for measurement; contraindication to passive leg raising (PLR) including hip or spine surgery, intracranial hypertension and intra-aortic balloon pump support; and absence of an echocardiography examiner. We also excluded post-cardiac surgery patients because of lower IVC acquisition rate resulted from subcostal drainage and modified cardiac structure which prevented precise assessment of necessary parameters.

The study was conducted in compliance with the Declaration of Helsinki and was approved by the ethics committee of our institution. Informed consent was obtained from the next of kin.

\section{Echocardiography}

Echocardiograms were recorded within the first $24 \mathrm{~h}$ of ICU admission using an echocardiograph (CX50, PHILIPS, USA) with a $2.5-\mathrm{MHz}$ phased-array probe. Images were saved for offline analysis. Two intensivists who were experienced in echocardiography performed the echo examination. Electrocardiograms were recorded continuously during the echo examination. Three cardiac cycles were analyzed and averaged. M-mode and Doppler echocardiographic measurements were taken according to standard protocols.

The left ventricular ejection fraction (LVEF) was obtained using a modified biplane Simpson's method from apical two- and four-chamber views. The mitral annular plane systolic excursion (MAPSE) was obtained from the apical 4-chamber view by positioning the cursor along the lateral mitral ring and measuring the difference between the highest and lowest point of the M-mode sinusoid wave [17]. TAPSE was also obtained from the apical 4-chamber view by positioning the $\mathrm{M}$-mode cursor along the lateral part of the tricuspid valve ring [18]. The LVOT velocity-time integral (VTI) was obtained from pulsed Doppler imaging by positioning the sample 
volume at the LVOT approximately $0.5 \mathrm{~cm}$ below the aortic valve [19]. The diameter of the IVC was measured in M-mode through the subcostal longitudinal plane, just upstream of the origin of the suprahepatic vein. The patients were all in controlled ventilation and the diameter of IVC was measured using M-mode. dIVC was calculated as (maximum diameter on inspiration - minimum diameter on expiration)/minimum diameter on expiration [3].

\section{Passive leg raising manoeuvre}

PLR is considered a reliable method of predicting volume responsiveness, even in spontaneously breathing patients $[20,21]$. In a recent meta-analysis, the area-under-thecurve (AUC) of PLR for predicting fluid responsiveness in patients with shock could be up to 0.95 [22]. The PLR manoeuvre was performed by first placing the patient in a semi-recumbent position with the head elevated at $45^{\circ}$ and then positioning the patient supine with the legs straight and elevated at $45^{\circ}$ for $2 \mathrm{~min}$. The VTI was measured before PLR and after $90 \mathrm{~s}$ of PLR. We managed to obtain an optimal VTI spectrum within $120 \mathrm{~s}$ [23].

\section{Other parameters collected}

Demographic information, Acute Physiology and Chronic Health Evaluation (APACHE) II scores, Sequential Organ Failure Assessment (SOFA) scores, reasons for admission, currently used vasoactive agents and ICU mortality were collected for all patients. We also recorded each patient's heart rate (HR), mean arterial pressure (MAP) and ventilator settings at the time of the echo examination.

\section{Definition}

RV dysfunction was defined as TAPSE below $16 \mathrm{~mm}$ [13]. LV dysfunction was defined as LVEF below $50 \%$, as in prior studies [24, 25]. ILVD patients were defined as those with only LV systolic dysfunction (TAPSE $\geq 16 \mathrm{~mm}$, LVEF $<50 \%$ ). Fluid responsiveness was defined as a $10 \%$ increase in VTI after PLR $[9,21,26]$.

\section{Statistical analysis}

Statistical analysis was performed using the statistical software package SPSS 13.0 (SPSS, Inc., Chicago, Illinois, USA). Continuous data were expressed as the mean $\pm S D$ or the median and the interquartile range. Categorical variables were presented as frequency and percentages. The distributions of the continuous values were assessed for normality by the Kolmogorov-Smirnov test. Group comparisons were performed by Student's $t$ test, the Mann-Whitney $U$ test, the Chi-squared test, or Fisher's exact test where appropriate. Spearman's correlation coefficients and their corresponding $p$ values were

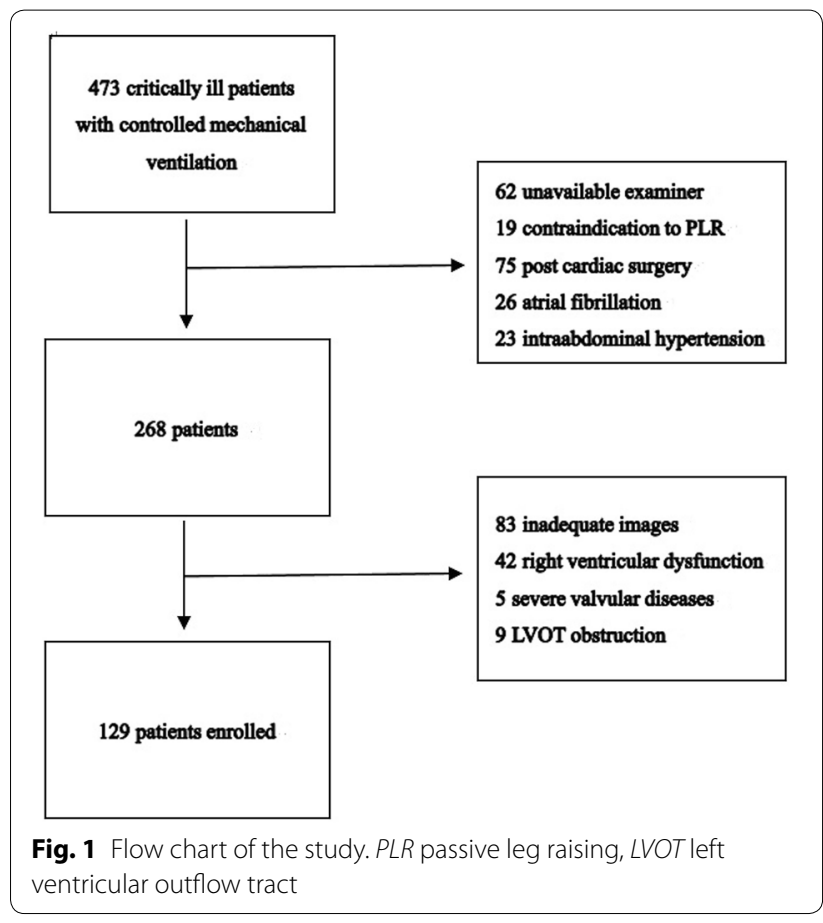

calculated to assess the variable relationships. Receiveroperating characteristic (ROC) curves were generated and the AUCs were then calculated and compared for all patients, patients with normal left ventricular function (NLVF) and ILVD groups. Intraobserver and interobserver variabilities in TAPSE, dIVC, LVEF, and VTI were assessed in 20 randomly selected patients and were tested using both paired $t$ tests and intraclass correlation coefficients (ICCs). An ICC $>0.8$ was considered excellent agreement. All $p$ values were two tailed and were considered significant for $p<0.05$.

\section{Results \\ Measurement variability}

The intraobserver variabilities in TAPSE, dIVC, LVEF, and VTI were minimal. The interobserver variability analysis revealed that ICCs for TAPSE, dIVC, LVEF, and VTI were: 0.967 (95\% CI 0.918-0.987), 0.940 (95\% CI $0.850-0.976), 0.900$ (95\% CI $0.748-0.960)$, and 0.953 (95\% CI 0.877-0.982), respectively.

\section{General characteristics of all patients}

A total of 473 patients were screened for enrolment. Three hundred and forty-four patients were excluded because of unavailability of an examiner, diagnoses that could be confounding factors, contraindication to PLR and inadequate images (Fig. 1). One hundred and twentynine patients were enrolled in this study, and the general characteristics are illustrated in Table 1 . The mean age of 
Table 1 General characteristics

\begin{tabular}{|c|c|}
\hline Categories & Findings $(n=129)$ \\
\hline Age (year) & $59 \pm 19$ \\
\hline Sex (male, \%) & $74(57.4 \%)$ \\
\hline APACHEII & $16 \pm 6$ \\
\hline SOFA & $6 \pm 4$ \\
\hline \multicolumn{2}{|l|}{ Reason for admission ( $n, \%)$} \\
\hline Noncardiac surgery & $61(47.2 \%)$ \\
\hline Circulatory shock & $49(38.0 \%)$ \\
\hline Respiratory failure & $13(10.1 \%)$ \\
\hline Others $^{\mathrm{a}}$ & $6(4.7 \%)$ \\
\hline \multicolumn{2}{|l|}{ Type of shock $(n, \%)$} \\
\hline Septic shock & $28(21.7 \%)$ \\
\hline Cardiogenic shock & $11(8.5 \%)$ \\
\hline Haemorrhagic shock & $5(3.9 \%)$ \\
\hline Other types & $5(3.9 \%)$ \\
\hline Tidal Volume (ml/kg) & $6.7(6.3,7.3)$ \\
\hline $\operatorname{PEEP}\left(\mathrm{cmH}_{2} \mathrm{O}\right)$ & $5.5 \pm 1.7$ \\
\hline NE infusion $(n, \%)$ & $55(42.6 \%)$ \\
\hline NE dose ( $\mu \mathrm{g} / \mathrm{kg} / \mathrm{min})$ & $0.2(0.1,0.35)$ \\
\hline Lactate $(\mathrm{mmol} / \mathrm{L})$ & $2.7(1.9,3.5)$ \\
\hline Timing of echo (hour from admission) & $10(5,20)$ \\
\hline Volume administered before examination ( $\mathrm{ml}$ ) & $1595(800,2462)$ \\
\hline \multicolumn{2}{|l|}{ Prognosis } \\
\hline ICU mortality $(n, \%)$ & $16(12.4 \%)$ \\
\hline
\end{tabular}

APACHE acute physiology and chronic health evaluation, SOFA sequential organ failure assessment, $P E E P$ positive end-expiratory pressure, NE norepinephrine, CVP central venous pressure, ICU intensive care unit

a Diabetic ketoacidosis, cerebral disease and kidney failure

the subjects was 59 years, and $57.4 \%$ were men. The mean APACHE II and SOFA scores were $16 \pm 6$ and $6 \pm 4$, respectively. The reasons for admission included noncardiac surgery (47.2\%), various types of shock (38.0\%), respiratory failure (10.1\%), and other reasons $(4.7 \%)$, i.e., diabetic ketoacidosis, cerebral disease, and kidney failure. The median echo examination timing was at $10 \mathrm{~h}$ after ICU admission. The median volume administered before echo was $1595 \mathrm{ml}$. The ICU mortality was $12.4 \%$.

\section{Haemodynamic and echocardiographic parameters of the patients}

According to the echocardiographic results, 28 patients displayed ILVD and the remaining 101 patients who displayed normal biventricular functions were labelled as NLVF patients. The HR and MAP were similar in these two groups of patients. The TASPE in the two groups were not significant different $(20.8 \pm 3.4 \mathrm{~mm}$ vs. $22.1 \pm 3.7 \mathrm{~mm}, p=0.111)$. The MAPSE and LVEF in ILVD patients were lower $(11.9 \pm 3.8 \mathrm{~mm}$ vs. $14.9 \pm 3.3 \mathrm{~mm}$, $p<0.001$ and $38 \%$ vs. $65 \%, p<0.001)$. The ILVD patients had lower baseline VTI $(p=0.025)$. The two groups had
Table 2 Hemodynamic and echocardiographic parameters of the patients

\begin{tabular}{lllr}
\hline Categories & ILVD $(\boldsymbol{n}=\mathbf{2 8})$ & NLVF $(\boldsymbol{n}=\mathbf{1 0 1})$ & \multicolumn{1}{c}{$\boldsymbol{p}$} \\
\hline HR $(\mathrm{bpm})$ & $91 \pm 18$ & $88 \pm 19$ & 0.325 \\
MAP $(\mathrm{mmHg})$ & $78 \pm 13$ & $79 \pm 15$ & 0.695 \\
MAPSE $(\mathrm{mm})$ & $11.3 \pm 2.8$ & $14.9 \pm 3.3$ & $<0.001$ \\
TAPSE $(\mathrm{mm})$ & $20.8 \pm 3.4$ & $22.1 \pm 3.7$ & 0.111 \\
LVEF $(\%)$ & $38(29,45)$ & $65(60,71)$ & $<0.001$ \\
IVCEE $(\mathrm{mm})$ & $14.9 \pm 3.4$ & $15.8 \pm 3.8$ & 0.278 \\
dIVC (\%) & $20(13,24)$ & $16(6,25)$ & 0.211 \\
VTI (cm) & $17.7 \pm 5.1$ & $20.1 \pm 4.9$ & 0.025 \\
VTI post PLR (cm) & $18.0 \pm 5.0$ & $21.6 \pm 4.8$ & 0.003 \\
Number of PLR & $5(17.9 \%)$ & $57(56.4 \%)$ & $<0.001$ \\
$\quad$ responders $(n, \%)$ & & & \\
\hline
\end{tabular}

ILVD isolated left ventricular systolic dysfunction, NLVF normal left ventricular function, $H R$ heart rate, MAP mean arterial pressure, VTI velocity-time integral, $P L R$ passive leg raising, IVCEE diameter of inferior vena cava at end expiration, MAPSE mitral annular plane systolic excursion, TAPSE tricuspid annular plane systolic excursion, LVEF left ventricle ejection fraction, dIVC inferior vena cava distensibility index, MRLF mismatch of right and left heart function, PLR passive leg raising

similar end-expiratory IVC diameters $(14.9 \pm 3.4 \mathrm{~mm}$ vs. $15.8 \pm 3.8 \mathrm{~cm}, p=0.278$ ).

The ILVD patients had dIVC value as high as that in NLVF patients ( $20 \%$ vs. $16 \%, p=0.211)$, while the ILVD group had a much lower proportion of PLR responders than the NLVF group $(17.9 \%$ vs. $53.2 \%, p<0.001)$ (Table 2, Fig. 2a, b).

\section{Correlation analysis of dIVC and $\Delta \mathrm{VTI}$}

dIVC was associated with $\Delta$ VTI among all patients, $r=0.535, p<0.001$. No correlation between those two variables was found in ILVD patients, $r=0.196, p=0.309$. When we specifically examined NLVF patients, dIVC was still associated with $\Delta \mathrm{VTI}$ in that group, $r=0.722$, $p<0.001$, and the correlation was strengthened, $Z=-2.336, p=0.020$ (Fig. 3a-c).

\section{ROC analysis of dIVC for the detection of fluid responsiveness}

To evaluate the sensitivity and specificity of dIVC for assessing fluid responsiveness, we generated ROC curves. The ROC analysis showed that the AUC of dIVC for determining fluid responsiveness was 0.815 in all patients $(95 \%$ CI $0.742-0.889 ; p<0.001$ ). In patients with ILVD, the AUC of dIVC for determining fluid responsiveness was 0.550 ( $95 \%$ CI $0.283-0.817 ; p=0.729$ ). After the ILVD patients were excluded, i.e., in NLVF group, the ROC analysis revealed an AUC of 0.918 (95\% CI $0.858-$ 0.978; $p<0.001$ ), which was significantly different from the AUC derived from all patients, $Z=2.134, p=0.033$ (Table 3, Fig. 4a-c). 


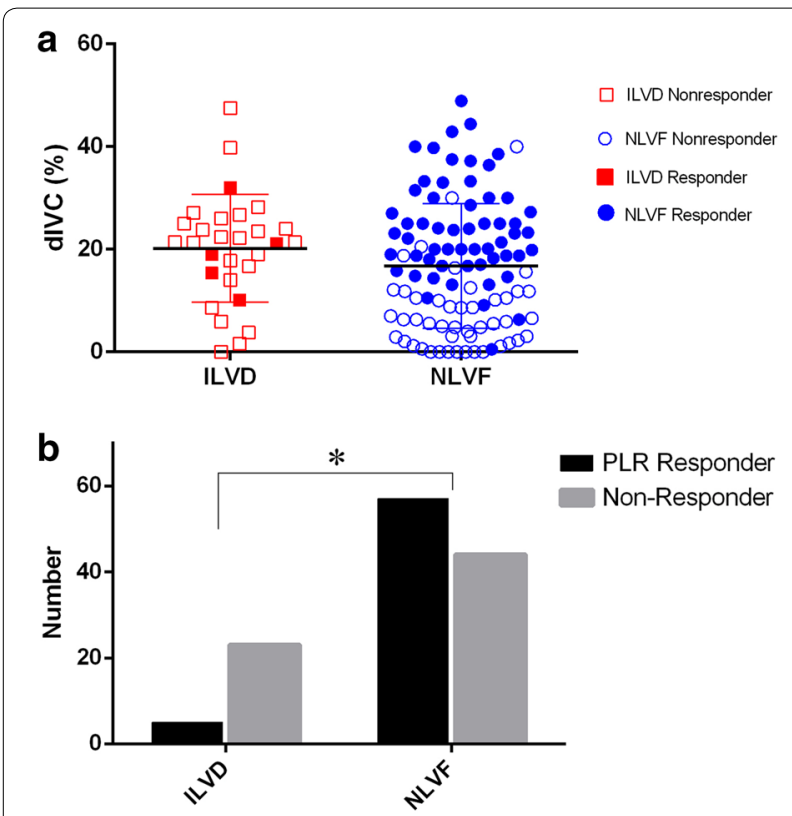

Fig. 2 Distribution of dIVC and proportion of PLR responders in ILVD and NLVF patients. a There was no difference in dIVC value between ILVD and NLVF patients, $p=0.211$. $\mathbf{b}$ ILVD patients had much lower proportion of PLR responders than that in NLVF patients, $p<0.001$. ILVD isolated left ventricular systolic dysfunction, NLVF normal left ventricular function, dIVC inferior vena cava distensibility index, $P L R$ passive leg raising

\section{Analysis of patients with different LVEF levels}

We divided all the patients into those with LVEF below 40\%, LVEF $40-50 \%$, and LVEF above $50 \%$. dIVC was not statistically different among patients with different LVEF levels $(p=0.247)$. Patients with LVEF below $40 \%$ had the lowest $\triangle \mathrm{VTI}$ and fewest patients with PLR response $(p<0.001)$ (Table 4).

\section{Discussion}

In this study, we investigated the use of dIVC for fluid responsiveness assessment in critically ill patients on controlled mechanical ventilation. We demonstrated that when there was an isolated LV systolic dysfunction, i.e., normal RV function in combination with impaired LV function, the effectiveness of dIVC for predicting fluid responsiveness was compromised.

We opted to use PLR to assess fluid responsiveness, because fluid challenge provides a risk of volume overload in a high proportion of critically ill patients. The effectiveness of PLR has been validated by other researchers [27]. The differences in the cutoff value could be due to different samples, different criteria for stroke volume increase, or even the use of different equations to calculate dIVC $[3,4]$. We chose TAPSE to represent RV
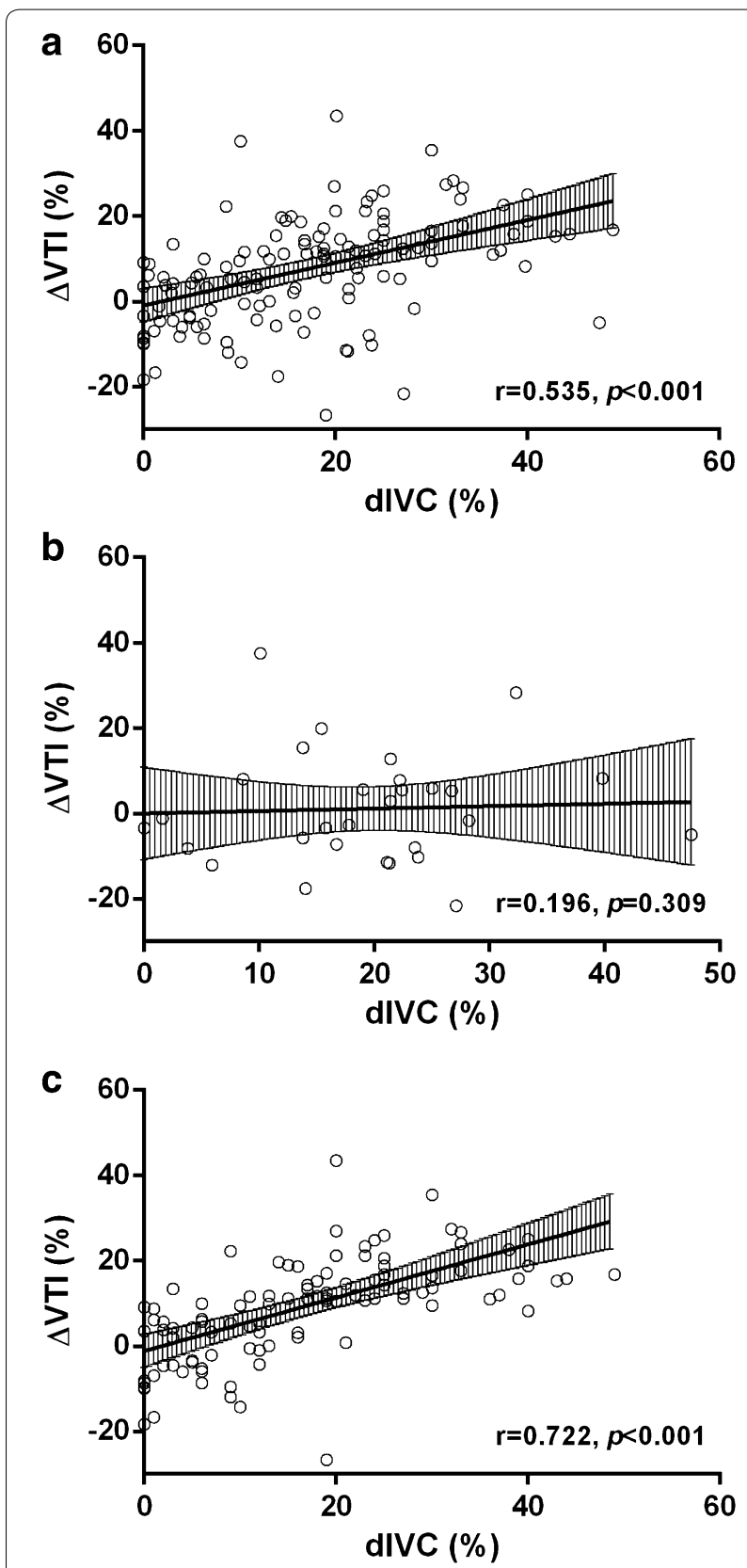

Fig. 3 Correlation of dIVC and $\triangle \mathrm{VTI}$. a Correlation of dIVC and $\triangle \mathrm{VTI}$ in all patients, $r=0.535, p<0.001$. b Correlation of dIVC and $\triangle V T I$ in ILVD patients, $r=0.196, p=0.309$. c Correlation of dIVC and $\triangle V T$ I in NLVF patients, $r=0.722, p<0.001$. Correlation of dIVC and $\triangle V T I$ derived from NLVF patients was strengthened than that derived from all patients, $p=0.020$. ILVD isolated left ventricular systolic dysfunction, NLVF normal left ventricular function; dIVC inferior vena cava distensibility index, $\triangle V T /$ velocity-time integral change after passive leg raising

function on the basis that TAPSE is easy to measure, less operator dependent and is correlated with the biplane Simpson RV EF and myocardial performance index [28]. 
Table 3 dIVC for the detecting of fluid responsiveness

\begin{tabular}{|c|c|c|c|c|c|c|c|c|}
\hline Categories & AUC & $95 \% \mathrm{Cl}$ & $p$ & $\begin{array}{l}\text { Optimum } \\
\text { cutoff (\%) }\end{array}$ & Sen (\%) & Spe (\%) & PPV (\%) & NPV (\%) \\
\hline All patients $(n=129)$ & 0.815 & $0.742-0.889$ & $<0.001$ & 16.5 & 79.0 & 72.1 & 72.4 & 78.7 \\
\hline $\operatorname{NLVF}(n=101)^{\mathrm{a}}$ & 0.918 & $0.858-0.978$ & $<0.001$ & 14.5 & 82.7 & 87.0 & 89.7 & 84.5 \\
\hline $\operatorname{ILVD}(n=28)$ & 0.550 & $0.283-0.817$ & 0.729 & 21.5 & 40.0 & 58.3 & 17.3 & 81.7 \\
\hline
\end{tabular}

NLVF normal left ventricular function, ILVD isolated left ventricular systolic dysfunction, dIVC inferior vena cava distensibility index, $A U C$ area-under-the-curve, $C I$ confidence interval, Sen sensitivity, Spe specificity, PPV positive predictive value, NPV negative predictive value

a In comparison with AUC derived from all patients, $p=0.033$

The present study showed that ILVD is not uncommon in critically ill patients. After excluding patients with RV dysfunction, patients with normal RV function and LV dysfunction still accounted for $21 \%$ of our study sample. Chockalingam et al. identified the common causes of acute left ventricular dysfunction in critically ill patients, including acute coronary syndrome (ACS), takotsubo cardiomyopathy, and global hypokinesis induced by sepsis or other insults [29]. The aforementioned diagnosis frequently results in abnormal LVEF, but rather not necessarily result in concomitant RV dysfunction [30-33]. Thus, ILVD is not rare in ICU. Although we were unable to collect enough information about the patients' LV function status before ICU admission, some patients might have preexisting LV dysfunction. In addition, we believe that some acute conditions also account for this phenomenon, such as newly onset of stressed cardiomyopathy or septic cardiomyopathy. Pulido et al. performed echo examinations of 68 severe sepsis and septic shock patients. The frequency of LV systolic dysfunction was $42.6 \%$ and only approximately half of the patients had RV dysfunction simultaneously [33].

This study revealed that there was no correlation between dIVC and $\triangle$ VTI in patients with ILVD. We speculated that it was the mismatch of RV and LV function that led to this result. Normally, the output of the right and left ventricle is the same and a patient would respond to fluid loading only when both ventricles operate on the ascending portion of the Frank-Starling curve. If one of the ventricles operates on the flat portion of the curve, the cardiac output will not increase significantly in response to volume expansion [35]. The notion that IVC variation could reflect fluid responsiveness is also based on the theory that the two ventricles usually have equal function. If the left ventricle is impaired first, the right ventricle usually becomes involved as well, which is because the underlying injury may affect both ventricles, or the two ventricles may affect each other through ventricular interdependence. However, in ILVD patients, the function of the two ventricles might become incompatible and respond differently to preload increase, and high dIVC might merely reflect a potential stroke volume increase in the RV and not the LV [12, 34]. Therefore, the mismatch between the biventricular functions in ILVD patients could diminish the accuracy of volume status assessment of IVC. In this study, we excluded patients with RV dysfunction, because IVC was not able to accurately reflect volume status in this situation. For patients with RV dysfunction such as chronic pulmonary hypertension, RV infarction or severe tricuspid regurgitation, fluid responsiveness may still exist, while the IVC diameter is large and its variation is small [11]. However, when the RV and LV function are both impaired, whether IVC can reflect fluid responsiveness need further investigation.

We found that the ILVD patients had higher IIVC than NLVF patients, though not statistically different. IVC reflects the interaction of venous return and RV function. The physicians had various ways to understand the patients' heart function including medical history, physical examination, or even cardiac ultrasound. They might choose more conservative strategy in terms of volume administration in patients with LV dysfunction.

Fluid responsiveness is one of the key steps in haemodynamic management [36]. The echocardiographic plane of the IVC is easily obtained at the bedside and is less dependent on image quality than other echocardiographic imaging parameters. With an increasing number of ICU physicians being trained in focused cardiac ultrasound examination, IVC variation are used increasingly often in the management of critically ill patients [37-39]. Nonetheless, for ILVD patients, if fluid was administered according to the value of dIVC, the chance of a stroke volume increase would very slim. Fluid therapy guided solely by dIVC would put the patient at risk of occurrence or exacerbation of pulmonary oedema. Therefore, ILVD should be taken into account when the IVC is used to predict fluid responsiveness. In addition to assessing IVC variation before administering fluid, one should simultaneously examine heart function, which could improve clinical decision making and lower the risk of volume overload.

We also assessed the difference of patients with mildly depressed LV function and those with markedly 
Fig. 4 ROC analysis of dIVC for the detection of fluid responsiveness. a Area-under-the-curve (AUC) of dIVC for the detection of fluid responsiveness in all patients 0.815 (95\% Cl 0.742-0.889; $p<0.001)$. b In ILVD patients, the AUC was only 0.550 ( $95 \% \mathrm{Cl} 0.283-0.817 ; p=0.729$ ). c In NLVF patients, the ROC analysis revealed an AUC of $0.918(95 \% \mathrm{Cl}$ $0.858-0.978 ; p<0.001$ ), which was statistically significant compared with the AUC derived from all patients, $p=0.033$. ILVD isolated left ventricular systolic dysfunction, NLVF normal left ventricular function; dIVC inferior vena cava distensibility index

low LVEF. The results showed that the latter group was less likely to respond to volume expansion. Rather than diagnosing patients as LV dysfunction, the exact LVEF value should be taken into consideration when treating ILVD patients. Our results were in line with a recent study focusing on hemodynamic type of septic shock [40]. They discovered that septic shock patients with LV systolic dysfunction had a median LVEF of $29 \%$. Therefore, although LVEF below 50\% was the cutoff value for LV dysfunction, a lower LVEF seemed to be more clinically relevant.

\section{Limitations}

This study has several limitations. First, this study was conducted at a single centre, and the enrolled population was heterogeneous. We were unable to provide the exact aetiologies for ILVD, which can result from ACS or coronary arterial disease, takotsubo cardiomyopathy, septic cardiomyopathy, or other causes. However, the enrolment heterogeneity and lack of an aetiology would not prevent the deduction of the conclusion, which is based on the echocardiographic appraisal of heart function. Second, although the patients with intra-abdominal hypertension before the PLR test were excluded, we did not know the real value of intra-abdominal pressure during PLR. As elevated intra-abdominal pressure can impede PLR-induced venous return, it might result in false negatives of this manoeuvre [41]. Third, we chose to use LVEF, an afterload-dependent parameter, as the marker of LV dysfunction. Other parameters such as tissue Doppler or strain would be more appropriate than LVEF for reflecting the intrinsic contractility of the left ventricle. However, those markers are more machine- or operator dependent than LVEF. Finally, we did not include patients with biventricular dysfunction in this study, and we failed to differentiate patients with LV diastolic dysfunction from patients with a normal LVEF. The above-mentioned diagnoses also could be confounding factors affecting the utility of IVC

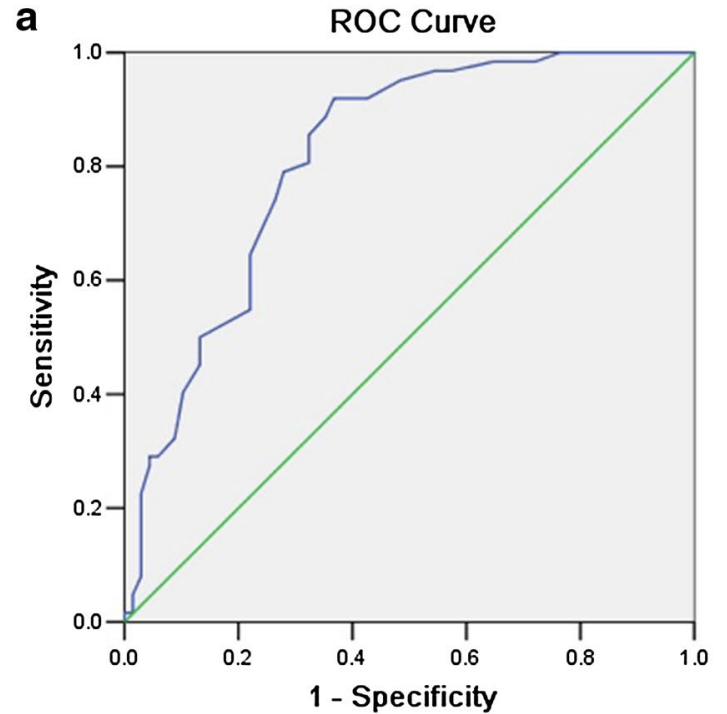

b
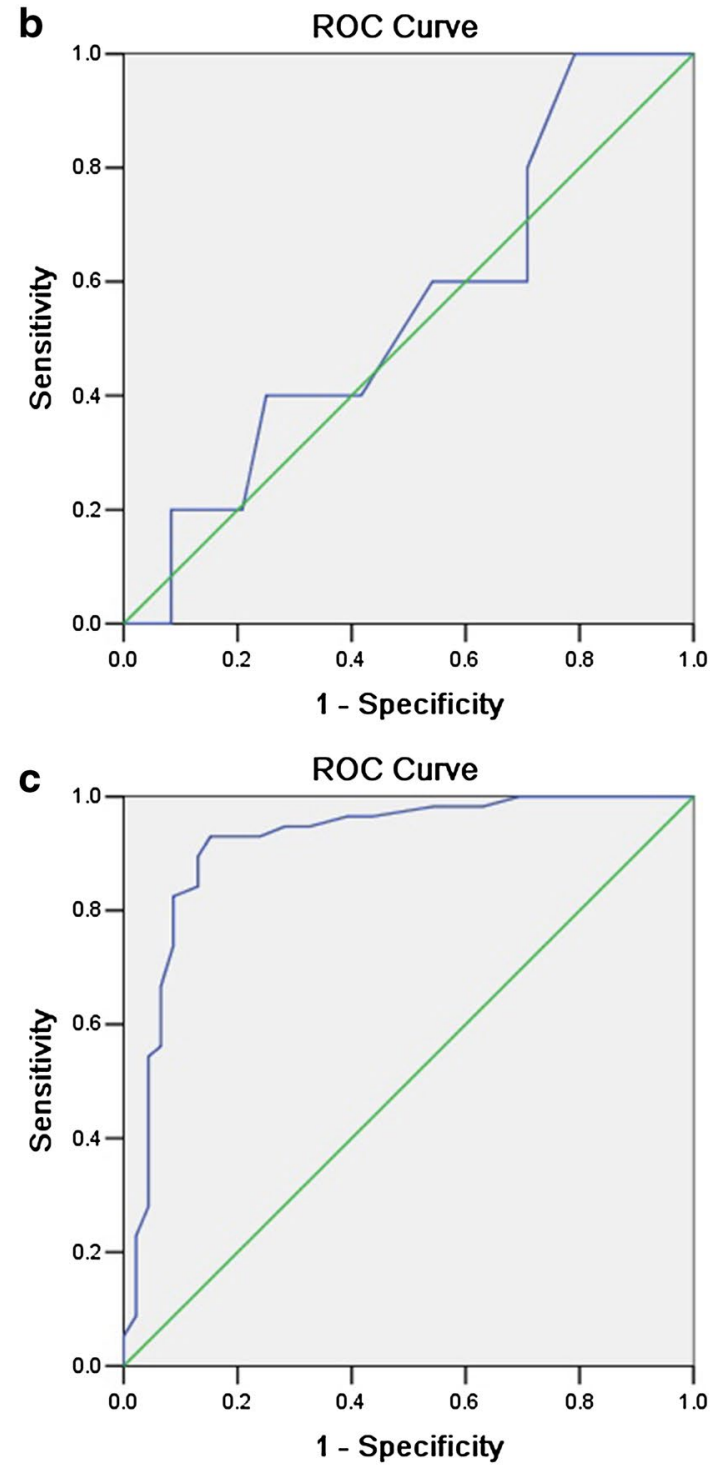
Table 4 dIVC and $\Delta V T I$ in patients with different LVEF

\begin{tabular}{|c|c|c|c|c|}
\hline Categories & LVEF $>50 \%(n=101)$ & $\begin{array}{l}\text { LVEF } 40-50 \% \\
(n=11)\end{array}$ & $\begin{array}{l}\text { LVEF }<40 \% \\
(n=17)\end{array}$ & $p$ \\
\hline dIVC (\%) & $16(6,25)$ & $19(15,22)$ & $20(12,26)$ & 0.247 \\
\hline$\Delta \mathrm{VTI}(\%)$ & $10.8(2.0,16.6)$ & $5.5(-1.3,18.1)$ & $-2.9(-9.8,3.3)$ & $<0.001$ \\
\hline Number of PLR responders ( $n, \%)$ & 57 (56.4\%) & $4(36.4 \%)$ & $1(5.9 \%)$ & $<0.001$ \\
\hline
\end{tabular}

dIVC inferior vena cava distensibility index, VTI velocity-time integral, LVEF left ventricle ejection fraction, $P L R$ passive leg raising

effectiveness for fluid responsiveness assessment, and future studies are warranted.

\section{Conclusions}

Mismatch between RV and LV function should be taken into consideration in assessing the fluid responsiveness of ICU patients through IVC variation. dIVC should be used with caution when critically ill patients on controlled mechanical ventilation display normal right heart function in combination with abnormal left heart systolic function.

\begin{abstract}
Abbreviations
ILVD: isolated left ventricular systolic function; NLVF: normal left ventricular function; IVC: inferior vena cava; dIVC: IVC distensibility index; TAPSE: tricuspid annular plane systolic excursion; LVEF: left ventricular ejection fraction; VTI: velocity-time integral; ICU: intensive care unit; RV: right ventricle; ACP: acute cor pulmonale; LVOT: left ventricular outflow tract; MAPSE: mitral annular plane systolic excursion; PLR: passive leg raising; APACHE: acute physiology and chronic health evaluation; SOFA: sequential organ failure assessment; HR: heart rate; MAP: mean arterial pressure; CVP: central venous pressure; ROC: receiver-operating characteristic; ICC: intraclass correlation coefficient; PEEP: positive end-expiratory pressure; AUC: area-under-the-curve; PPV: positive predictive value; NPV: negative predictive value; ACS: acute coronary syndrome; AMl: acute myocardial infarction.
\end{abstract}

\section{Acknowledgements}

This study was performed at Critical Care Department of Peking Union Medical College Hospital.

We would like to thank Dr. Wei He, from Beijing Tongren Hospital, for his good advice on the study design. Chengli Shen, from Division of Surgical Oncology, the Ohio State University Wexner Medical Centre for his kind suggestions on the statistical issue.

\section{Authors' contributions}

$\mathrm{HZ}$ designed the study, analyzed and interpreted data, performed the statistical analysis, and drafted the manuscript. QZ obtained data and revised manuscript. XC analyzed data and revised the manuscript; XW analyzed data and revised the manuscript. DL conceived and designed the study, interpreted data and revised the manuscript. All authors read and approved the final manuscript.

\section{Funding}

None.

\section{Availability of data and materials}

All data sets used and analyzed during the current study are available from the corresponding author on reasonable request.

\section{Ethics approval and consent to participate}

This study was approved by the ethics committee of Peking Union Medical College Hospital, Beijing, China (Approval No. ZS-1602). Written informed consent was obtained from the next of kin of each patient.

\section{Consent for publication}

Not applicable.

\section{Competing interests}

The authors declare that they have no competing interests.

\section{Author details}

${ }^{1}$ Department of Critical Care Medicine, Peking Union Medical College Hospital, Chinese Academy of Medical Sciences and Peking Union Medical College, Beijing, China. ${ }^{2}$ Pittsburgh Heart, Lung, Blood and Vascular Institute, University of Pittsburgh, School of Medicine, Pittsburgh, PA, USA. ${ }^{3}$ Department of Critical Care Medicine, Peking Union Medical College Hospital, Chinese Academy of Medical Sciences, 1 \# Shuai Fu Yuan, Dong Cheng District, Beijing 100730, China.

Received: 28 April 2019 Accepted: 26 September 2019

Published online: 07 October 2019

\section{References}

1. Michard F, Teboul JL. Predicting fluid responsiveness in ICU patients: a critical analysis of the evidence. Chest. 2002;121(6):2000-8.

2. Vincent JL, Sakr Y, Sprung CL, Ranieri VM, Reinhart K, Gerlach H, et al. Sepsis in European intensive care units: results of the SOAP study. Crit Care Med. 2006;34(2):344-53.

3. Barbier C, Loubières Y, Schmit C, Hayon J, Ricôme JL, Jardin F, et al. Respiratory changes in inferior vena cava diameter are helpful in predicting fluid responsiveness in ventilated septic patients. Intensive Care Med. 2004;30(9):1740-6.

4. Feissel M, Michard F, Faller JP, Teboul JL. The respiratory variation in inferior vena cava diameter as a guide to fluid therapy. Intensive Care Med. 2004;30(9):1834-7.

5. Huang H, Shen Q, Liu Y, Xu H, Fang Y. Value of variation index of inferior vena cava diameter in predicting fluid responsiveness in patients with circulatory shock receiving mechanical ventilation: a systematic review and meta-analysis. Crit Care. 2018;22(1):204.

6. Orso D, Paoli I, Piani T, Cilenti FL, Cristiani L, Guglielmo N. Accuracy of ultrasonographic measurements of inferior vena cava to determine fluid responsiveness: a systematic review and meta-analysis. J Intensive Care Med. 2018. https://doi.org/10.1177/0885066617752308.

7. Si X, Xu H, Liu Z, Wu J, Cao D, Chen J, et al. Does respiratory variation in inferior vena cava diameter predict fluid responsiveness in mechanically ventilated patients? A systematic review and meta-analysis. Anesth Analg. 2018;127(5):1157-64.

8. Cavaliere F, Cina A, Biasucci D, Costa R, Soave M, Gargaruti R, et al. Sonographic assessment of abdominal vein dimensional and hemodynamic changes induced in human volunteers by a model of abdominal hypertension. Crit Care Med. 2011;39(2):344-8.

9. Vieillard-Baron A, Evrard B, Repessé X, Maizel J, Jacob C, Goudelin M, et al. Limited value of end-expiratory inferior vena cava diameter to predict fluid responsiveness impact of intra-abdominal pressure. Intensive Care Med. 2018;44(2):197-203.

10. Mandelbaum A, Ritz E. Vena cava diameter measurement for estimation of dry weight in haemodialysis patients. Nephrol Dial Transpl. 1996;11(Suppl 2):24-7. 
11. Via G, Tavazzi G, Price S. Ten situations where inferior vena cava ultrasound may fail to accurately predict fluid responsiveness: a physiologically based point of view. Intensive Care Med. 2016;42(7):1164-7.

12. Maclver DH, Clark AL. The vital role of the right ventricle in the pathogenesis of acute pulmonary edema. Am J Cardiol. 2015;115(7):992-1000.

13. Rudski LG, Lai WW, Afilalo J, Hua L, Handschumacher MD, Chandrasekaran $\mathrm{K}$, et al. Guidelines for the echocardiographic assessment of the right heart in adults: a report from the American Society of Echocardiography endorsed by the European Association of Echocardiography, a registered branch of the European Society of Cardiology, and the Canadian Society of Echocardiography. J Am Soc Echocardiogr. 2010;23(7):685-713 (quiz 786-8).

14. Vieillard-Baron A. Assessment of right ventricular function. Curr Opin Crit Care. 2009;15(3):254-60.

15. Slama M, Tribouilloy C, Maizel J. Left ventricular outflow tract obstruction in ICU patients. Curr Opin Crit Care. 2016;22(3):260-6.

16. Blaser A, Malbrain ML, Starkopf J, Fruhwald S, Jakob SM, De Waele J, et al. Gastrointestinal function in intensive care patients: terminology, definitions and management Recommendations of the ESICM Working Group on Abdominal Problems. Intensive Care Med. 2012;38(3):384-94.

17. Sarti A, Poli C, Marchiani S. The left ventricle. In: Sarti A, Lorini L, editors. Echocardiography for intensivists. Italia: Springer-Verlag; 2009. p. 75-90.

18. Forfia PR, Fisher MR, Mathai SC, Housten-Harris T, Hemnes AR, Borlaug BA, et al. Tricuspid annular displacement predicts survival in pulmonary hypertension. Am J Respir Crit Care Med. 2006;174(9):1034-41.

19. Quiñones MA, Otto CM, Stoddard M, Waggoner A, Zoghbi WA. Recommendations for quantification of Doppler echocardiography: a report from the Doppler Quantification Task Force of the Nomenclature and Standards Committee of the American Society of Echocardiography. J Am Soc Echocardiogr. 2002;15(2):167-84.

20. Teboul JL, Monnet X. Prediction of volume responsiveness in critically ill patients with spontaneous breathing activity. Curr Opin Crit Care. 2008;14(3):334-9.

21. Cavallaro F, Sandroni C, Marano C, La Torre G, Mannocci A, De Waure C, et al. Diagnostic accuracy of passive leg raising for prediction of fluid responsiveness in adults: systematic review and meta-analysis of clinical studies. Intensive Care Med. 2010;36(9):1475-83.

22. Cherpanath TG, Hirsch A, Geerts BF, Lagrand WK, Leeflang MM, Schultz $\mathrm{MJ}$, et al. Predicting fluid responsiveness by passive leg raising: a systematic review and meta-analysis of 23 clinical trials. Crit Care Med. 2016;44(5):981-91.

23. Maizel J, Airapetian N, Lorne E, Tribouilloy C, Massy Z, Slama M. Diagnosis of central hypovolemia by using passive leg raising. Intensive Care Med. 2007;33(7):1133-8.

24. Vieillard-Baron A, Caille V, Charron C, Belliard G, Page B, Jardin F. Actual incidence of global left ventricular hypokinesia in adult septic shock. Crit Care Med. 2008;36(6):1701-6.

25. Parrillo JE. Pathogenetic mechanisms of septic shock. N Engl J Med. 1993;328(20):1471-7

26. Monnet X, Rienzo M, Osman D, Anguel N, Richard C, Pinsky MR, et al. Passive leg raising predicts fluid responsiveness in the critically ill. Crit Care Med. 2006;34(5):1402-7.
27. Geri G, Vignon P, Aubry A, Fedou AL, Charron C, Silva S, et al. Comparison of echocardiographic indices used to predict fluid responsiveness in ventilated patients. Am J Respir Crit Care Med. 2017;195(8):1022-32.

28. Miller D, Farah MG, Liner A, Fox K, Schluchter M, Hoit BD. The relation between quantitative right ventricular ejection fraction and indices of tricuspid annular motion and myocardial performance. J Am Soc Echocardiogr. 2004;17(5):443-7.

29. Chockalingam A, Mehra A, Dorairajan S, Dellsperger KC. Acute left ventricular dysfunction in the critically ill. Chest. 2010;138(1):198-207.

30. Bajaj A, Sethi A, Rathor P, Suppogu N, Sethi A. Acute Complications of Myocardial Infarction in the Current Era: diagnosis and Management. J Investig Med. 2015;63(7):844-55.

31. Khera R, Light-McGroary K, Zahr F, Horwitz PA, Girotra S. Trends in hospitalization for takotsubo cardiomyopathy in the United States. Am Heart J. 2016;172:53-63.

32. Park JH, Kang SJ, Song JK, Kim HK, Lim CM, Kang DH, et al. Left ventricular apical ballooning due to severe physical stress in patients admitted to the medical ICU. Chest. 2005;128(1):296-302.

33. Pulido JN, Afessa B, Masaki M, Yuasa T, Gillespie S, Herasevich V, et al. Clinical spectrum, frequency, and significance of myocardial dysfunction in severe sepsis and septic shock. Mayo Clin Proc. 2012;87(7):620-8.

34. Campbell P, Drazner MH, Kato M, Lakdawala N, Palardy M, Nohria A, et al. Mismatch of right- and left-sided filling pressures in chronic heart failure. J Card Fail. 2011;17(7):561-8.

35. Michard F, Teboul JL. Using heart-lung interactions to assess fluid responsiveness during mechanical ventilation. Crit Care. 2000;4(5):282-9.

36. Dellinger RP, Levy MM, Rhodes A, Annane D, Gerlach H, Opal SM, et al. Surviving Sepsis Campaign: international guidelines for management of severe sepsis and septic shock, 2012. Intensive Care Med. 2013;39(2):165-228.

37. Sekiguchi H, Harada Y, Villarraga HR, Mankad SV, Gajic O. Focused cardiac ultrasound in the early resuscitation of severe sepsis and septic shock: a prospective pilot study. J Anesth. 2017;31(4):487-93.

38. Blanco P, Miralles Aguiar F, Vallejo A. Point-of-care ultrasonography in critical care medicine: a one way directional road. J Ultrasound. 2016;19(2):157-8.

39. Greenstein YY, Littauer R, Narasimhan M, Mayo PH, Koenig SJ. Effectiveness of a Critical Care Ultrasonography Course. Chest. 2017;151(1):34-40.

40. Geri G, Vignon P, Aubry A, Fedou AL, Charron C, Silva S, et al. Cardiovascular clusters in septic shock combining clinical and echocardiographic parameters: a post hoc analysis. Intensive Care Med. 2019;45(5):657-67.

41. Beurton A, Teboul JL, Girotto V, Galarza L, Anguel N, Richard C, et al. IntraAbdominal hypertension is responsible for false negatives to the passive leg raising test. Crit Care Med. 2019;47(8):e639-47.

\section{Publisher's Note}

Springer Nature remains neutral with regard to jurisdictional claims in published maps and institutional affiliations.

\section{Submit your manuscript to a SpringerOpen ${ }^{\odot}$ journal and benefit from:}

- Convenient online submission

- Rigorous peer review

- Open access: articles freely available online

- High visibility within the field

- Retaining the copyright to your article

Submit your next manuscript at springeropen.com 